\title{
Star Formation in Isolated LIRGs: Clues to Star-forming Processes at Higher z
}

\author{
Isaura Fuentes-Carrera ${ }^{1}$, Lorenzo Olguín ${ }^{2}$, Patricia Ambrocio-Cruz ${ }^{3}$, \\ Simon Verley ${ }^{4}$, Margarita Rosado $^{5}$, Lourdes Verdes-Montenegro ${ }^{6}$, \\ Paolo Repetto $^{5}$, Celia Vázquez ${ }^{1}$ and Verónica Aguilera ${ }^{1}$ \\ ${ }^{1}$ Escuela Superior de Física y Matemáticas, Instituto Politécnico Nacional \\ U.P. Adolfo López Mateos, Col. Zacatenco \\ México, D.F., C.P. 07730 Mexico \\ email: isaura@esfm.inp.mx, isaura.fuentescarrera@gmail.com \\ ${ }^{2}$ Departamento de Investigación en Física, Universidad de Sonora \\ Sonora, Mexico \\ email: lorenzo@astro.uson.mx \\ ${ }^{3}$ Ciencias de la Tierra y Materiales, Universidad Autónoma del Estado de Hidalgo \\ Hidalgo, Mexico \\ email: silviap@uaeh.edu.mx \\ ${ }^{4}$ Deptartamento de Física Teórica y del Cosmos, Universidad de Granada \\ Granada, Spain \\ email: simon@ugr.es \\ ${ }^{5}$ Instituto de Astronomía, Universidad Nacional Autónoma de México \\ México, D.F., Mexico \\ email: margarit@astroscu.unam.mx,prepetto@astro.unam.mx \\ ${ }^{6}$ Instituto de Astrofísica de Granada (IAA/CSIC) \\ Granada, Spain \\ email: lourdes@iaa.es
}

\begin{abstract}
Luminous infrared galaxies (LIRGs) are galaxies with $\mathrm{L}_{I R}>10^{11} \mathrm{~L}_{\odot}$. For a starforming galaxy to emit at a LIRG level, it must have a very high star formation rate (SFR). In the local Universe, the star formation (SF) is primarily triggered by interactions. However, at intermediate redshift, a large fraction of LIRGs are disk galaxies with little sign of recent merger activity. The question arises whether the intermediate redshift LIRGs are "triggered" or experiencing "normal", if elevated, SF. Understanding these SF processes is important since this type of systems may have contributed to $20 \%$ or more of the cosmic SFR in the early Universe. In order to address this issue we study similar systems in the Local Universe, that is isolated late-type galaxies displaying LIRG activity. We use different observational techniques in order to trace the star-forming history of these systems. Here we present preliminary results.
\end{abstract}

Keywords. galaxies: kinematics and dynamics, galaxies: starburst, galaxies: interactions

\section{Introduction}

Luminous infrared galaxies (LIRGs) are galaxies with total infrared (IR) luminosity between $10^{11} L_{\odot}$ and $10^{12} L_{\odot}$ (Sanders \& Mirabel 1996). The high IR luminosities of LIRGs result from thermal dust heating in starbursts (SBs), or active galactic nuclei (AGN). Dust absorbs optical and ultraviolet radiation, and reemits that energy in a broad thermal peak in the mid to far IR $(8$ to $1000 \mu \mathrm{m})$. In order for a star forming galaxy to emit at a LIRG level, it must have a very high star formation rate (SFR), in excess of $\sim 17 M_{\odot} y r^{-1}$ (Melbourne et al. 2007). In the local Universe, these high SFR are primarily triggered by galaxy-galaxy interactions or mergers (Ishida 2004). Local LIRGs 


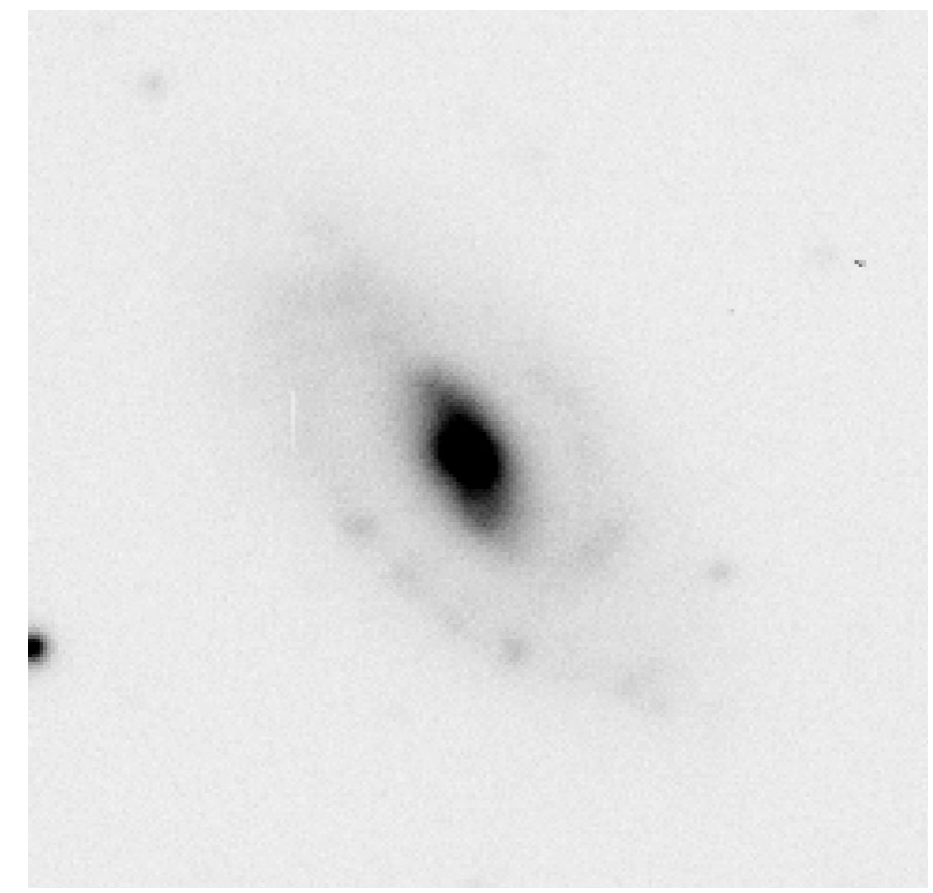

Figure 1. H $\alpha$ image of CIG 866 taken with the $1.5 \mathrm{~m}$ telescope in the Observatorio Astronómico Nacional, San Pedro Mártir (OAN-SPM) in Baja California, Mexico. North to the top, east to the left. FoV is $60 "$ x $60 "$ ". Continuum has not been subtracted.

(along with ULIRGs, $\mathrm{L}_{I R}>10^{12} L_{\odot}$ ) are known to be predominantly triggered by major mergers with the star formation and/or AGN activity triggered by gas compression and inflows towards the center (Sanders \& Mirabel 1996; Borne et al. 2000). This is in good agreement with galaxy evolution models (e.g., Mihos \& Hernquist 1996; Springel, Di Matteo \& Hernquist (2005)), which also suggest that galaxies must be in the throes of a major merger to funnel sufficient gas towards the bottom of the gravitational potential of the merging system. LIRGs and ULIRGs have long been recognized as one of the best laboratories to study the processess of violent star formation in the Local Universe.

However, several authors show that roughly $50 \%$ of intermediate redshift LIRGs are disk galaxies with little sign of recent merger activity (Bell et al. 2005; Melbourne, Koo \& Le Floc'h (2005); Lotz et al. 2008). Marcillac et al. (2006) show that a fraction of the on-going star formation in the form of LIRGs takes place in less-perturbed systems, while Zheng et al. (2004) find that more than 1/3 of LIRGs with star-formation rates higher than $100 \mathrm{M}_{\odot} \mathrm{yr}^{-1}$ in fact have disk-like geometries, while only $6 \%$ show the obvious signs of major mergers -see also Elbaz \& Cesarsky (2003). While morphology rules out major mergers as triggers for the majority of intermediate redshift LIRGs, interactions with neighbors, minor mergers, or bar instabilities may contribute. Bell et al.(2005) suggest that infrared activity in distant LIRGs may be related to higher gas masses in isolated spirals, which perhaps experience gas infall, or to minor mergers, where the smaller component is not detected. It is currently an open question, whether the intermediate redshift LIRGs are "triggered" or experiencing "normal", if elevated, SF.

From a cosmological perspective, this type of SF is far from being negligible, since the co-moving number densities of infrared-luminous galaxies at $\mathrm{z} \sim 2$ indicate that these systems may have contributed to $20 \%$ or more of the cosmic star-formation rate in the 

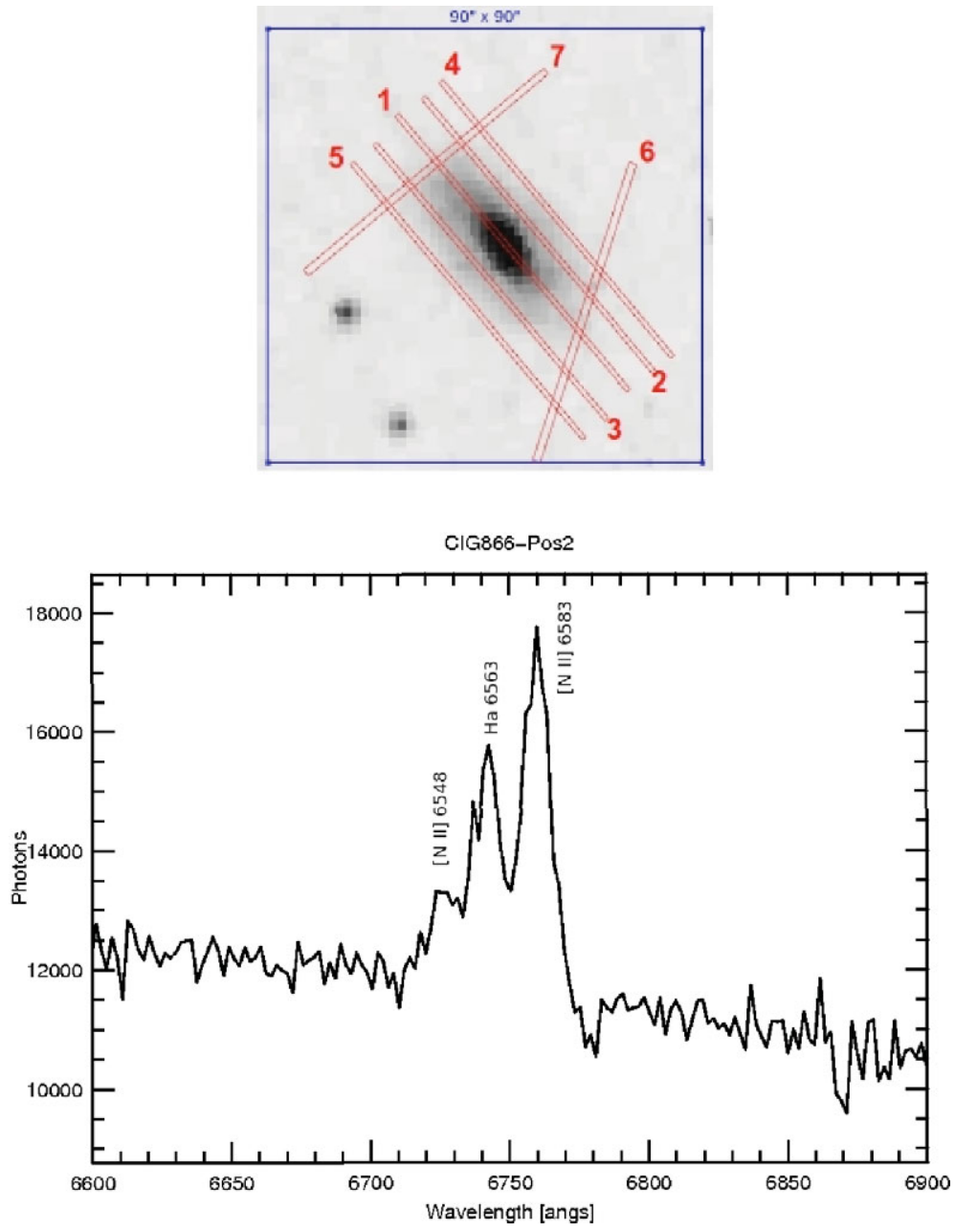

Figure 2. Top panel: Direct DSS image of CIG 866 indicating different slit positions. Bottom panel: Spectrum correposponding to slit number 2. Observations were done on the $2.1 \mathrm{~m}$ telescope in the OAN-SPM using the Boller \& Chivens spectrometer.

early Universe (Blain \& Phillips (2002)). In order to address this issue we present a study of similar systems in the Local Universe by analyzing the star-formation history ( $\mathrm{SFH})$ of isolated LIRGS. Direct imaging, long-slit spectroscopy and extended scanning FabryPerot spectroscopy are used to map the SF regions and underlying stellar population in these galaxies, as well as gas motions involved in the SF processes.

\section{Isolated LIRGs}

Galaxies considered for this work have been taken from the AMIGA sample (VerdesMontenegro et al. 2005). The AMIGA project (Analysis of the Interstellar Medium of Isolated Galaxies) aims to build and parametrize a statistically significant control sample of the most isolated galaxies in the Local Universe. It involves a refinement of the pioneering Catalog of Isolated Galaxies, CIG (Karachentseva 1973). The two largest refinements include uniform reevaluation of morphology (Sulentic et al. 2005) and isolation degree (Verley et al. 2007a; Verley et al. 2007b). This last point is of great importance 
for this work, since we want to guarantee there are no nearby companions perturbing these galaxies and thus inducing SF through tidal perturbations or torques. None of these galaxies seems to harbor any AGN, which indicates the excess in IR luminosity is solely due to very enhanced star-forming activity. The isolation criteria used to select our LIRG sample take into account the local number density of neighbor galaxies and the tidal strength that these produce on the candidate isolated galaxy. When a galaxy shows low values for both parameters, this galaxy can be considered very isolated form any sort of external influence.

\section{Preliminary Results}

Figure 1 shows the $\mathrm{H} \alpha$ image of the isolated LIRG CIG 866. This image shows faint HII regions in the southern part of the galaxy. Very diffuse emission is seen in the northern parts of the galaxy. Bottom panel of Figure 2 shows the long-slit spectrum of a region in the Sc galaxy CIG 866. This region is indicated with number 2 in the top panel of the same Figure. Though this is a rough spectrum, we can see that [NII] at $6583 \AA$ seems to be brighter than $\mathrm{H} \alpha$ which could be indicative of on-going shocks in these regions and probable violent SF. The extended kinematics of these regions, derived with the scanning Fabry-Perot interferometer PUMA (Rosado et al. 1995) need to be analyzed in order to explore the see if any important non-circular motions are present in these regions supporting the scenario of violent star-forming processes. The combination of long-slit information from different regions of the galaxy and the extended kinematics of the ionized gas, along with the direct imaging of the galaxy will tell us if we are witnessing a recent merging event in which CIG 866 "swallowed" a smaller galaxy -such as a satellite- or if this galaxy is hosting a bar -which is hinted in the optical imagesthus supporting the scenario proposed by Bell et al. (2005).

\section{References}

Bell, E. F., Papovich, C., Wolf, C., Le Floc'h, E., Caldwell, J. A. R. et al. 2005. ApJ, 625, 23 Blain, A. W. \& Phillips, T.G. 2002 MNRAS, 333, 222

Borne, K. D., Bushouse, H., Lucas, R. A. \& Colina, L. 2000 ApJ, 529L, 77

Elbaz, D. \& Cesarsky, C. 2003 Science, 300, 270

Ishida, C. M. 2004 PhD Thesis

Karachentseva, V. E. 1973, AIBSAO, 8, 3

Lotz, J. M., Jonsson, P., Cox, T. J. \& Primack, J. R. 2008 MNRAS 391, 1137

Marcillac, D., Elbaz, D., Charlot, S., Liang, Y. C., Hammer, F. et al. 2006 A $\& A, 458,369$

Melbourne, J., Koo, D. C. \& Le Floc'h, E. 2005 ApJ, 632, 65

Melbourne, J., Phillips, A. C., Harker, J., Novak, G. et al. 2007 ApJ, 660, 81

Mihos, J. C. \& Hernquist, L. 1996 ApJ 464, 641

Rosado, M., Langarica, R., Bernal, A., Cobos, F., Garfias, F. et al. 1995 RMxAC, 3, 263

Sanders, D. B. \& Mirabel, F. 1996, ARAESA, 34, 749

Springer, V., Di Matteo, T. \& Hernquist, L. 2005 MNRAS, 361, 776

Sulentic, J., Verdes-Montenegro, L., Bergond, G., Lisenfeld, U. et al. 2005 A $\& A, 449,937$

Verdes-Montenegro, L., Sulentic, J., Lisenfeld, U., Leon, S., Espada, D., Garcia, E., Sabater, J., \& Verley, S. 2005, A\& $A, 436,443$

Verley, S., Odewahn, S.C., Verdes-Montenegro, L., Leon, S. et al. 2007 A\&SA, 470, 505

Verley, S., Leon, S., Verdes-Montenegro, L., Combes, F., Sabater, J. et al. 2007 A $\& A$ 472, 121

Zheng, X. Z., Hammer, F., Flores, H., Assémat, F. \& Pelat, D. 2004 A\&A, 421, 847 